\title{
La Relación del Estrés con el Desempeño Laboral del Personal de Agro Rural
}

\author{
The Relationship of Stress with The Labor Performance of \\ Agro Rural Staff
}

\section{RESUMEN}

El Dr. Francisco Becerra, Subdirector de la Organización Panamericana de la Salud (OPS) en el año 2016 mencionó: Hoy el mundo del trabajo con sus avances industriales, la globalización, el desarrollo tecnológico y las comunicaciones virtuales, nos impone retos y condiciones que con frecuencia exceden los límites de nuestras habilidades y capacidades. Su resultado es el estrés, que puede conducirnos a disfunciones físicas, mentales y sociales; mermar nuestra productividad y afectar nuestros círculos familiares y sociales, incluso dañar nuestra salud. Cuando cualquier persona tiene un problema y no puede solucionarlo, su cuerpo comienza a verse afectado tanto física como mentalmente, las primeras manifestaciones son la depresión, dolores musculares, pérdida del apetito, pérdida del interés y la concentración, entre otras. También el estrés induce ataques cardíacos, eleva la presión sanguínea, genera dolores de cabeza y aumenta la presión cardíaca y respiratoria. En el ámbito laboral, el estrés produce que el trabajador disminuya su compromiso con la organización donde labora, así como su rendimiento de desempeño laboral, causa que el trabajador se ausente, rote de puestos, renuncie, etc. Lo que afecta directamente en las organizaciones haciéndolas incompetentes, baja la productividad, y afecta su imagen pública. Por todas estas razones, decidí analizar la relación del estrés con el desempeño laboral de los trabajadores de la Sede Central Lima de Agro Rural, ya que su labor es amplia y su trabajo extenuante, porque ellos coordinan con los especialistas de las diferentes gerencias departamentales del país las acciones técnicas y administrativas para la programación, ejecución, seguimiento, supervisión y evaluación de proyectos a su cargo, todo ello llevado por constante presión por parte de sus superiores. Palabras claves: Estrés; Desempeño Laboral; Personal.

\section{ABSTRACT}

Dr. Francisco Becerra, Deputy Director of the Pan American Health Organization (PAHO) in 2016 mentioned: Today the world of work with its industrial advances, globalization, technological development and virtual communications, imposes challenges and conditions that They often exceed the limits of our skills and abilities. Its result is stress, which can lead
Katia Sujey Rojas Arancibia

katiarojas7@hotmail.com

Universidad Nacional del Altiplano 
to physical, mental and social dysfunctions; reduce our productivity and affect our family and social circles, even damage our health. When anyone has a problem and cannot solve it, their body begins to be affected both physically and mentally, the first manifestations are depression, muscle aches, loss of appetite, loss of interest and concentration, among others. Stress also induces heart attacks, raises blood pressure, generates headaches and increases heart and respiratory pressure. In the workplace, stress causes the worker to decrease his commitment to the organization where he works, as well as his performance of work performance, causes the worker to be absent, job rotation, resignation, etc. What directly affects organizations making them incompetent, lowers productivity, and affects their public image. For all these reasons, I decided to analyze the relationship of stress with the work performance of the workers of the Lima Headquarters of Agro Rural, since their work is extensive and their work is exhausting, because they coordinate with the specialists of the different departmental management of the country technical and administrative actions for the programming, execution, monitoring, supervision and evaluation of projects under their charge, all this carried out by constant pressure from their superiors.

Keywords: Stress; Job performance; Personal.

\section{INTRODUCCIÓN}

Uno de los problemas actuales que afectan el desempeño laboral del trabajador es el estrés, que afecta tanto su salud como la realización de sus funciones.

$\mathrm{Al}$ considerar al estrés puede referirse a una condición negativa o una condición positiva que responda a un factor estresante y que pueda tener un impacto en la salud mental o física y en el bienestar de una persona. Hoy en día, la salud está reconocida como una combinación de factores biológicos, psicológicos (pensamientos, emociones y comportamientos), y sociales (socioeconómicos, socio ambientales, y culturales). (Organización Internacional del Trabajo, 2016, p.2)

Por otro lado, el desempeño laboral según Dessler y Varela (2017) lo definen como cualquier procedimiento que incluya el establecimiento de factores o estándares laborales y se evalúe el desempeño real de los empleados en relación con esos estándares.

En el Perú de acuerdo a un estudio del Instituto de Opinión Pública de la Pontificia Universidad Católica del Perú (PUCP) publicado en el diario La República el 28 de Marzo del 2018 , señala que el $80 \%$ de peruanos sufren o han sufrido estrés, lo que ha conllevado a cam- bios frecuentes en su estado de ánimo (23.8\%), mostrar sueño durante el día (22.4\%), insomnio (21.3\%), depresión (15.6\%). Las causas del estrés están centralizadas en las excesivas demandas que existen en el trabajo tanto en tareas como en el tiempo y los efectos en el individuo son muchos ya que desgasta al organismo, el bienestar y la salud. En este tipo de situaciones el rendimiento y la productividad disminuyen; se afecta la calidad de las relaciones interpersonales y el desarrollo psicosocial y profesional. (Oblitas, 2017)

La institución analizada es el Programa de Desarrollo Productivo Agrario Rural - Agro Rural, una unidad ejecutora del Ministerio de Agricultura y Riego que depende del Viceministerio de Desarrollo e Infraestructura Agraria y Riego y cuya finalidad es promover el desarrollo agrario rural a través del financiamiento de programas rurales, proyectos de inversión pública y actividades en zonas rurales del ámbito agrario, y articular las acciones en territorios de menor grado de desarrollo económico.

Se observa que en dicha institución existe un absentismo y abandono de sus trabajadores, al realizar el consolidado de los Indicadores de Personal de Agro Rural, comprobé una disminución de su personal desde el año 2014 hasta el año 2016 en casi un 20\%, equivalente a 236 trabajadores. Otro aspecto observado fue que 
no se ejecuta todo el presupuesto programado, realizando el consolidado de los Indicadores de Resultados de Agro Rural comprobé que no se ejecutaron al $100 \%$ los presupuestos de los años 2014 al 2016.

Por todo lo antes mencionado, el presente artículo tuvo como objetivo general determinar de qué manera se relaciona el Estrés con el Desempeño Laboral del personal del Programa de Desarrollo Productivo Agrario Rural - Agro Rural sede central Lima - 2017.

\section{MÉTODOS}

El estudio fue observacional, de corte transversal, descriptivo, correlacional, realizado el 2017, la población estuvo conformada por 54 trabajadores de la Sede Central Lima de Agro Rural, la técnica fue la encuesta, el instrumento fue el Cuestionario.

El porcentaje del nivel de estrés, y de desempeño laboral de los trabajadores de Agro Rural Sede Central Lima se obtuvo con la tabulación y suma de los ítems o preguntas del cuestionario, cada ítem de los 20, contó con 5 alternativas de respuesta: Nunca, ocasionalmente, algunas veces, frecuentemente y siempre, identificados de 1 á 5 acordes a la escala de Likert, el nivel de estrés y de desempeño laboral contó con 3 niveles de medición basados en los puntajes finales de todos los ítems (Bajo, medio y alto). El análisis de las variables se realizó en forma descriptiva, considerando las variables para las causas del estrés a: volumen y ritmo de trabajo, tareas monótonas aburridas y triviales, nivel de responsabilidad, condiciones ambientales del lugar de trabajo, inestabilidad laboral, reconocimiento laboral, abuso de poder político, acoso laboral, aumento de la edad de jubilación y falta de conciliación entre la vida familiar y la vida laboral. Y las variables del desempeño laboral se consideró a: Calificación para el puesto, motivación en el trabajo, orientación hacia el trabajo, madurez en el trabajo y trabajo de equipo e interacción; para identificar la frecuencia se tabularon los datos del cuestionario pregunta por pregunta para así conocer las cantidades de toda la muestra. En cuanto a la relación de variables, los datos obtenidos resultaron del uso del software SPSS, y para relacionar las variables estudiadas: Causas del estrés y Factores de desempeño laboral, se apli- có la prueba no paramétrica, de correlación de datos cualitativos Chi cuadrado, con el 95\% de confiabilidad y significancia del $\mathrm{p}<0.05$.

\section{RESULTADOS}

El personal de Agro Rural de la Sede Central Lima, estuvo comprendido por varones (57\%), personas de entre 31 y 50 años (55\%), casados (48\%), personal contratado (63\%).

Se identificó las causas del Estrés del personal de la sede Central Lima de Agro Rural: Volumen y ritmo de trabajo (75\%), Inestabilidad Laboral (72\%), Aumento de la edad de jubilación (72\%), Abuso de poder político (61\%), Nivel de Responsabilidad (59\%), Reconocimiento laboral (59\%), Tareas monótonas, aburridas y triviales (52\%), Falta de conciliación entre la vida laboral y familiar (42\%), Condiciones ambientales del lugar de trabajo (32\%), Acoso Laboral (29\%).

Se identificó los factores de Desempeño Laboral del personal de la sede Central Lima de Agro Rural: Motivación (52\%), Orientación hacia el trabajo (46\%), Calificación para el puesto (43\%), Trabajo en equipo e interacción (42\%) y Madurez en el trabajo (39\%).

Finalmente, al relacionar las variables causas del estrés (Volumen y ritmo de trabajo) se demostró que, si existe relación estadísticamente significativa a un $95 \%$ de confianza, con el factor de desempeño laboral (Motivación) por ser la proporción menor a 0.05 .

\section{DISCUSION}

Para efectos de la realización de este artículo, las Causas del Estrés que actualmente son conocidos como Factores de Riesgos Psicosociales se adaptaron de: (Cox, et al., 2000), y de la (Organización Internacional del Trabajo, 2016), concluyendo que los factores de riesgo psicosocial son aquellos aspectos del contenido de trabajo y contexto del trabajo, y resultan ser causantes de dañar al trabajador psicológicamente y físicamente.

1. Contenido del Trabajo, se refiere a las causas del estrés relacionado con las condiciones de trabajo y la organización del trabajo. El contenido del trabajo incluye factores de riesgo como: el volumen y rit- 
mo de trabajo, tareas monótonas, aburridas y triviales, el nivel de responsabilidad y las condiciones ambientales del lugar de trabajo.

2. Contexto del Trabajo, hace referencia a las causas del estrés en la organización del trabajo y las relaciones laborales, tales como el reconocimiento laboral, el acoso laboral, la inestabilidad laboral, el abuso de poder político, la edad de jubilación, y la falta de conciliación entre la vida familiar y laboral.

\begin{tabular}{|l|}
\hline CONTENIDO DEL TRABAJO \\
\hline 1.1. La Sobrecarga y la Intensificación del Trabajo \\
\hline 1. El Volumen y ritmo de trabajo. \\
\hline 1.1.2. Las Tareas monótonas, aburridas y triviales. \\
\hline 1.2. Las Demandas Emocionales \\
\hline 1.2.1. El nivel de responsabilidad. \\
\hline 1.2.2. Las condiciones ambientales del lugar de trabajo. \\
\hline CONTEXTO DEL TRABAJO \\
\hline 2.1. La Inseguridad Laboral y el Acoso \\
\hline La Inestabilidad laboral. \\
\hline El Reconocimiento laboral. \\
\hline El Abuso de poder político. \\
\hline El Acoso laboral. \\
\hline 2.2. La Jubilación \\
\hline 2.2.1. El Aumento de la edad de jubilación. \\
\hline 2.3. La Conciliación entre vida la Familiar y la vida Laboral \\
\hline $\begin{array}{l}\text { 2.3.1. La Falta de conciliación entre la vida familiar y la vida } \\
\text { laboral. }\end{array}$ \\
\hline
\end{tabular}

Figura 1. Causas del Estrés - Factores de Riesgos Psicosociales. Fuente: Adaptado de Cox, Griffiths y Rial (2000); Organización Internacional del Trabajo (2016). Elaboración Propia.

\section{Contenido del Trabajo.}

Sobrecarga y la Intensificación del Trabajo.

Dentro de este aspecto tenemos:

$$
\text { El Volumen y ritmo de trabajo. }
$$

El volumen de trabajo se refiere a la sobrecarga en cantidad de las funciones que se debe realizar en el trabajo, y el ritmo de trabajo se refiere al aspecto cualitativo, es decir la dificultad del trabajo a desempeñar, ambos aspectos tienen efectos sobre la salud y están asociado con el estrés (Agencia Europea para la Seguridad y la Salud en Trabajo, 2005).
Las Agencias Zonales de Agro Rural son las encargadas de realizar acciones técnicas y administrativas para la programación, ejecución, seguimiento, supervisión y evaluación de proyectos a su cargo, éstas son laboriosas, en el caso particular de la sede central Lima la tensión es mayor porque ellos realizan la consolidación de todas las gerencias departamentales y agencias zonales del Perú.

\section{Las Tareas monótonas, aburridas y triviales.}

Producen aburrimiento o fatiga mental, ésta se debe a la realización de actividades repetitivas y sin atractivo, la falta de variedad en el desempeño del trabajo, trabajo fraccionado y la utilización por debajo de las capacidades. A veces ocasiona inquietud, tristeza, fastidio que nos deja sin energía ni motivación ninguna. El aburrimiento disminuye la eficiencia y crece la variabilidad en el ritmo de trabajo. (Instituto Nacional de Seguridad y Salud en el Trabajo, 2010). Permanecer mucho tiempo en un cargo conlleva a la mecanización de la tarea y no permite desarrollar la capacidad laboral de los trabajadores.

En Agro Rural una vez que conoces y aprendes el teje y maneje del trabajo, todo se hace de forma mecánica, una y otra vez. Se convierte en un círculo vicioso.

\section{Las Demandas Emocionales.}

Dentro de las demandas emocionales se ha considerado:

\section{El nivel de responsabilidad.}

La responsabilidad se define como la cualidad que poseen las personas, cuando ponen cuidado y atención en lo que hacen o deciden. (Asociación Americana de Psicología, 2010)

El nivel de responsabilidad ha sido identificado como fuente potencial de estrés cuando existe un conflicto de funciones o roles a desempeñar, dicho conflicto se produce cuando la persona debe desempeñar un cargo que va contra sus creencias, principios y valores, o cuando existe incompatibilidad en las diversas funciones que desempeña.

En este aspecto los trabajadores de Agro Rural se enfrentan a excesiva responsabilidad 
en el desempeño de funciones, ambigüedad y conflicto de funciones, tienen mucha responsabilidad sobre todo cuando hay que realizar ejecución de obras, entrega de herramientas, rendiciones de cuentas, por mencionar algunas. Las responsabilidades caen sobre todo en los que están en el área de Administración que son los responsables de la ejecución y rendición de cuentas, sobre todo al final de cada trimestre. trabajo

Las condiciones ambientales del lugar de

Son todas las circunstancias físicas en las que el empleado se encuentra cuando realiza sus funciones o tareas, en la organización. Es el ambiente físico que rodea al empleado mientras ocupa su cargo en la organización. (Aamodt, 2017)

Las malas condiciones físicas de trabajo, pueden afectar al estrés sufrido por los trabajadores y afecta su salud física y psicológica. Los riesgos principales dentro de este aspecto son: iluminación, ruido, temperatura, humedad, ventilación, diseño físico del lugar de trabajo, etc., (Warr, 1992)

Al analizar este punto, la Sede Central Lima de Agro Rural se encuentra ubicada en Jesús María, en un edificio de 8 pisos, con moderna infraestructura, que cuenta con 2 ascensores, y con todos los equipos básicos para realizar su trabajo, el lugar es apropiado cuenta con ventilación y buena luz y cada área está distribuida por pisos.

\section{Contexto del Trabajo.}

\section{La Inseguridad Laboral y el Acoso.}

En este punto tenemos:

\section{La Inestabilidad Laboral.}

Es el temor a ser despedido de su centro de trabajo en cualquier momento y/o realizar una jubilación anticipada obligatoria y esto constituye una fuente principal de estrés (Marshall, 1977).

Para el caso específico de Agro Rural los más vulnerables son los que están bajo la modalidad de Locación de Servicios (Recibos por Honorarios) y Contratos Administrativos de Servicios (CAS), que lo conforman más del $50 \%$ de trabajadores a nivel nacional.

\section{El Reconocimiento Laboral.}

El reconocimiento laboral es una fuente importante de satisfacción y desarrollo personal y también ayuda a que las personas puedan estar cómodos con el desempeño de su trabajo y con su cotidiano vivir. Cuando se reconoce el buen desempeño del trabajo satisface y estimula a los trabajadores, impulsándolos hacia el éxito de las empresas u organizaciones. (Agencia Europea para la Seguridad y la Salud en Trabajo, 2005).

En Agro Rural existen varios problemas en este aspecto (estancamiento profesional, promoción excesiva o insuficiente), los incentivos, reconocimientos y ascensos están supeditados a la política, por lo que en la mayoría de los casos de los trabajadores estos solo tratan de cumplir con su trabajo y no van más allá.

El incentivo muchas veces no tiene que ser económico, basta que el trabajador reciba un reconocimiento público para aumentar su nivel de satisfacción en el desempeño de sus funciones, ya que este contribuye a que los empleados se sientan a gusto trabajando. El apreciar la labor de los empleados es fundamental para su buen desempeño.

\section{El Abuso de poder político.}

Se da cuando el poder se ha ejercido desequilibradamente y a favor de quienes ostentan otros poderes en la sociedad. Este problema muchas veces es causado por la corrupción, que es una manifestación del poder político, y se da por la búsqueda del beneficio propio y no por el bien común; es el favoritismo para unos en prejuicios de otros. (Camacho, 2008).

Como se mencionó anteriormente en Agro Rural se da el favoritismo muy marcado por la influencia política, cada gobierno entrante siempre pone a su personal de confianza en altos cargos, los que a su vez se rodean de su círculo más cercano, dejando un mínimo porcentaje a aquellas personas capacitadas o preparadas para el cargo.

\section{El Acoso laboral.}

Está conformado por conductas de violencia psicológica de forma reiterada y prolongada en el marco de una relación laboral (Fidalgo, Galle- 
go, Ferrer, Nogareda, Pérez y García, 2010). Son ejemplos de acoso laboral: asignarles trabajos con plazos inalcanzables, sobrecargarle con mucho trabajo, quitarle responsabilidad, criticar continuamente su trabajo, ignorar sus éxitos o asignárselos a otras personas, etc. (Piñuel, 2013)

En Agro Rural el acoso laboral se da cuando desean sacar a algunos trabajadores sobre todo aquellos que no tienen vínculo laboral con ellos (CAS, Locación de Servicios), quizás porque algún recomendado desea tomar su lugar, eso los mantiene en constante zozobra.

\section{La Jubilación.}

\section{El Aumento de la edad de jubilación.}

Con el transcurrir del tiempo las personas sufren una disminución de las capacidades laborales, y su salud también se ve afectada. El aumento de las demandas laborales, las nuevas tecnologías y los cambios continuos en el trabajo dan lugar a que los trabajadores de mayor edad queden replegados. (Instituto Nacional de Seguridad y Salud en el Trabajo, 2010)

En el año 2018 los trabajadores que deseaban acceder a la jubilación ordinaria deberán tener una edad de 65 años y 6 meses, y hayan cotizado durante 36 años y 6 meses. Esto deja menos disfrute de la jubilación ya que con el paso de los años el cuerpo se deteriora y las enfermedades aumentan, y el dinero de jubilación que reciben cada vez es menor y no cubre sus necesidades básicas.

\section{La Conciliación entre la vida Familiar y la vida Laboral.}

La falta de Conciliación entre la vida Familiar y la vida Laboral.

El conflicto entre la vida familiar y la vida laboral se produce cuando los esfuerzos para cumplir con el rol del empleado interfieren con la capacidad de satisfacer las demandas del rol de cónyuge, progenitor o cuidador, también indicaron que la presión en el trabajo conlleva a mayor conflicto entre la vida familiar y la vida laboral (Demerouti, Bakker \& Bulters, 2004). Como resultado de esta disyuntiva, los hombres reducen su satisfacción familiar y las mujeres reducen su satisfacción laboral.
En definitiva, cuando el ámbito familiar de los trabajadores es afectado por el estrés, a consecuencia de la sobrecarga laboral esto influye en su estado de ánimo y en la manera como se relaciona en su familia (Byron, 2005).

\section{CONCLUSIONES}

1. Se identificó que la principal causa que provoca estrés en el personal de Agro Rural es el volumen y ritmo de trabajo que representa un $75 \%$ en los trabajadores de dicha institución, debido a que la Sede Central Lima realizar acciones técnicas y administrativas para la programación, ejecución, seguimiento, supervisión y evaluación de proyectos a su cargo, de todas las gerencias departamentales y agencias zonales del país, por ello la cantidad de trabajo es amplia, agotadora, estresante además se tiene que realizar en corto plazo. Esas extensas jornadas laborales provocan presión, tensión, fatiga o ansiedad en algunos casos.

2. Se identificó que el factor determinante en el desempeño Laboral del personal de Agro Rural, es la motivación que representa un $52 \%$ en los trabajadores de dicha institución, una explicación de esto sería que los trabajadores de Agro Rural debido a la constante presión por la cual ellos trabajan o están acostumbrados a trabajar la convierten en impulso o guía para alcanzar sus objetivos o logros en el desempeño de su trabajo.

3. Se determinó que el Personal del Programa de Desarrollo Productivo Agrario Rural - AGRO RURAL, sede central Lima 2017, ha obtenido un Nivel de Estrés Bajo.

De acuerdo a los resultados de la Encuesta realizada, los trabajadores de Agro Rural obtuvieron un Nivel de Estrés Bajo con $6 \%$, un Nivel de Estrés Medio con 83\% y un Nivel de Estrés Alto con $11 \%$.

5. Se determinó que el Personal del Programa de Desarrollo Productivo Agrario Rural - AGRO RURAL, sede central Lima 2017, ha obtenido un Nivel de Desempeño Laboral Medio. 
De acuerdo a los resultados de la Encuesta realizada, los trabajadores de Agro Rural obtuvieron un Nivel Desempeño Laboral Bajo con 15\%, Nivel de Desempeño Laboral Medio con 81\% y un Nivel de Desempeño Laboral Alto con 4\%.

\section{AGRADECIMIENTOS}

A la Escuela de Post Grado, Facultad de Ciencias Administrativas de la Universidad Nacional Mayor de San Marcos, al Director de la Unidad de Investigación, Dr. Fernando Valeriano, por su aporte valioso a este artículo; a los jefes de Agro Rural Sede Central Lima por dar viabilidad, participación y aporte a este artículo.

\section{REFERENCIAS BIBLIOGRÁFICAS}

Aamodt, M. (2012). Psicología industrial/organizacional: Un enfoque aplicado. Santa Fé, México: Cengage Leaning Editores.

Agencia Europea para la Seguridad y la Salud en Trabajo. (2005). Aportación de la Unión Europea a la seguridad y salud en las condiciones

Agro Rural. (2015). Manual de Operaciones. Recuperado de https://www.agrorural.gob.pe/ wp-content/uploads/transparencia/MORM-15-2015.pdf

Asociación Americana de Psicología. (2010). Los distintos tipos de estrés. Recuperado de https:// www.apa.org/centrodeapoyo/tipos

Camacho, D. (2008). Uso y abuso del poder político: (corrupción, crisis alimentaria, símbolos). Revista de Ciencias Sociales, 1(119), 1-3. Recuperado de https://www.redalyc.org/

Demerouti, E., Bakker, A.B., \& Bulters, A. (2004). The loss spiral of work pressure, workhome interference and exhaustion: Reciprocal relations in a three-wave study. Journal of Vocational Behaviour, 64, 131-149.

Dessler, G. y Varela, R.A. (2017). Administración de Recursos Humanos: Enfoque Latinoamericano. Estado de México, México: Pearson Educación.

Dinero. (2016, 1 de Diciembre). El estrés, "el mal del siglo XXI" que reduce la productividad de los empleados. Dinero. Recuperado de https:// www.dinero.com/internacional/articulo/banco-mundial-dice-que-el-estres-es-la-enfermedad-que-reduce-la-productividad/217965
Fidalgo M., Gallego Y., Ferrer R., Nogareda C., Pérez G. y García R. (2010). Acoso psicológico en el trabajo: definición. Madrid: Instituto Nacional de Seguridad e Higiene en el Trabajo.

Hernández R., Fernández C., y Baptista P. (2014). Metodología de la Investigación. Santa Fe, México: Mc Graw-Hill Interamericana Editores.

InfoJobs. (2018). Estado del Mercado laboral en España. Recuperado de https://nosotros.infojobs.net/wp-content/uploads/2018/05/ Informe-Anual-InfoJobs-ESADE-2017-Completo.pdf

Instituto Nacional de Seguridad y Salud en el Trabajo. (2010). El efecto sobre la salud de los riesgos psicosociales en el trabajo: una visión general. Recuperado de https://www.insst.es/InshtWeb/Contenidos/Instituto/Noticias/Noticias_ INSHT/2019/Ficheros/Efectos\%20sobre $\% 20$ la\%20salud\%20psicosociales.pdf

Marshall, J. (1977) Job pressures and satisfactions at managerial levels. Unpublished PhD thesis, University of Manchester Institute of Science and Technology, Manchester.

Organización Internacional del Trabajo. (2016). ESTRÉS EN EL TRABAJO: Un reto colectivo. Recuperado de https://www.ilo.org/wcmsp5/groups/ public/---ed_protect/---protrav/---safework/ documents/publication/wcms_466549.pdf

Organización Panamericana de la Salud. (2016). Estrés laboral es una carga para los individuos, los trabajadores y las sociedades. Recuperado de https://www.paho.org/

Piñuel, I. (2013). Mobbing, manual de autoayuda. Madrid, España: Ediciones Aguilar.

Redacciónlr. (28 de Marzo del 2018). El estrés, ese enemigo silencioso que afecta al $80 \%$ de peruanos. La República. Recuperado de https:// larepublica.pe/sociedad/1218645-el-estres-ese-enemigo-silencioso-que-afecta-al-80-de-peruanos/

RedacciónWapa. (21 de Junio del 2017). El 60\% de peruanos sufren de Estrés Laboral. Revista Wapa. Recuperado de https://wapa.pe/salud/1129856-el-60-de-peruanos-sufren-de-estres-laboral

Robbins, S., y Judge T. (2017). Comportamiento organizacional. Ciudad de México, México: Pearson Educación. 
